\title{
NOTAS SOBRE PROTOZOOS CILIADOS DULCEACUICOLAS DE CHILE
}

\section{NOTES ON CILIATED FRESHWATER PROTOZOA OF CHILE}

\author{
Stefan Woelfl \\ Instituto de Zoología, Universidad Austral de Chile, Valdivia \\ Casilla 567, Chile. Email: swoelfl@uach.cl
}

\begin{abstract}
RESUMEN
En este trabajo se revisa el estado de conocimientos sobre protozoos ciliados de aguas continentales en Chile. Por falta de especialistas, existen muy pocos estudios sobre la taxonomía, distribución geográfica y ecología de ciliados chilenos. Registros recientes existen solamente para los lagos araucanos, entre los cuales se describió Stentor araucanus, una especies endémica de Chile y Argentina. Se discuten problemas taxonómicos y se entregan detalles sobre la distribución de los géneros Stentor y Ophrydium en los lagos chilenos
\end{abstract}

Palabras Claves: Ciliados, biodiversidad, taxonomía, lagos chilenos.

\section{ABSTRACT}

This study reviews the state of knowlegde about ciliated protozoans in continental water bodies of Chile. Due to the lack specialists, exist very few studies on the taxonomy, the geographic distribution and the ecology of ciliates in Chile. Recent data exist only for the Araucanian Lakes, where with Stentor araucanus an edemic species of Chile and Argentina was described. Taxonomic problems are discussed and details about the distribution of the genus Stentor and Ophrydium in the chilean lakes are given.

KeYwords: Ciliates, biodiversity, taxonomy, Chilean lakes.

\section{INTRODUCCION}

Los ciliados son protozoos que se caracterizan por su alta diversidad, especialmente en aguas continentales, con alrededor de 1.125 géneros y más de 7.000 especies (Corliss 1979, Curds 1982). Aunque estos organismos juegan por lo general un rol clave en los ecosistemas acuáticos, rara vez han sido incluidos en estudios ecológicos de estos sistemas. Esto se debe principalmente por la falta de especialistas en la taxonomía del grupo y a la dificultad de determinar las especies. Por tal razón, existen muy pocos estudios sobre la taxonomía, distribución geográfica y ecología de ciliados en los cuerpos acuáticos continentales de Chile.
Se pueden distinguir tres épocas principales en las cuales se han estudiados los ciliados:

(a) 1902-1906: Estudios intensivos de ciliados en canales pequeños de Santiago (Bürger 1905, 1906), y descripción de algunos ciliados en estudios sobre los crustáceos del lago Villarrica (Daday 1902).

(b) 1953-1954: Descripción de algunos ciliados en estudios de crustáceos (Löffler 1961) y flora y fauna de los lagos araucanos (Thomasson 1963).

(c) 1990 - : Descripción de ciliados en estudios de lagos araucanos, con un enfoque ecológico (Foissner \& Woelfl 1994, Woelfl 1996, Geller et al. 1996, Woelfl \& Geller 2002). 
Protozoos ciliados de ecosistemas de DULCEACUÍCOLAS CHILENOS

Considerando solamente los registros recientes, que incluyen ciliados de los lagos araucanos (principalmente de los lagos Riñihue y Pirehueico), en la Tabla I se entrega un listado de los géneros y especies registradas. De estas especies se conoce solamente a Stentor araucanus como única taxa endémico en Chile (y Argentina) (Foissner \& Woelfl 1994).

Con respecto a la distribución de ciliados en lagos chilenos, existen registros más detallados sobre las especies $S$. amethystinus, $S$. araucanus y O. naumanni, sobre la base de información obtenida entre 1990 y 2004 (Woelfl \& Geller 2002, Woelfl sin publicar) (Tabla II). La distribución de la mayor parte de las restantes especies chilenas sigue siendo desconocida. Desde un punto de vista taxonómico, sólo existen problemas con las especies descritas por Bürger en 1905 y 1906. No se puede verificar la validez taxonómica de estas especias, ya sea por falta de material de referencia y/o por su descripción inapropiada. Estas especies corresponden a: Holostila aguaramdulium, Lacrimaria chilensis, Lacrimaria eliptica, Lacrimaria metabolica, Lacrimaria coniforme, Prorodon espejoci, Prorodon rigidus, Glaucoma paradoxa, Espejoia obtusa, Stegochilum acutum, Paramaecium nigrun y Strombidium armatum.

A nivel nacional, no existe ningún especialista en taxonomía de ciliados dulceacuícolas. Por esta razón, existe actualmente un vacío enorme de conocimiento sobre la taxonomía y distribución de ciliados en cuerpos acuáticos de Chile, especialmente en los extremos norte y sur del país. De igual manera, no existen colecciones de referencia de ciliados chilenos.

TABla I. Protozoos ciliados reportados en lagos araucanos durante 1990 - 2004 (según Foissner \& Woelfl, 1994; Woelfl, 1996; Woelfl \& Geller, 2002).

TABLE I. Ciliated protozoo recorded in the Araucanian lakes durind 1990 - 2004 (according to Foissner \& Woelfl, 1994; Woelfl, 1996; Woelfl \& Geller, 2002).

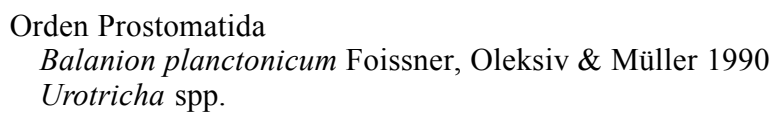

Orden Haptorida Askenasia spp.

Lacrymaria sp.

Orden Peritrichida Vorticella sp.

Ophrydium naumanni Pejler 1962

Vaginicola $\mathrm{sp.}$

Heterotrichida

Stentor amethystinus Leidy 1880

Stentor araucanus Foissner \& Woelfl 1994

Orden Oligotrichida

Strombidium spp.

Strobilidium spp.

Strobilidium viride Stein 1867

Halteria spp.

Orden Scuticociliatida

Cyclidium spp.

Uronema spp. 
Gayana 70(1), 2006

TABLA II. Ciliados recientemente encontrados en lagos araucanos (según Foissner \& Woelfl, 1994; Woelfl, 1996; Woelfl \& Geller, 2002).

TABLE II. Ciliated recently recorded in the Araucanian Lakes (according to Foissner \& Woelfl, 1994; Woelfl, 1996; Woelfl \& Geller, 2002).

\begin{tabular}{lccc}
\hline Lago & Stentor araucanus & Stentor amethystinus & $\begin{array}{c}\text { Ophrydium } \\
\text { naumanni }\end{array}$ \\
\hline Malleco & - & +++ & - \\
Galletué & + & - & - \\
Colico & +++ & +++ & + \\
Caburgua & +++ & +++ & + \\
Villarrica & + & + & + \\
Pellaifa & - & - & - \\
Pirehueico & +++ & +++ & - \\
Panguipulli & - & + & - \\
Riñihue & - & + & - \\
Neltume & - & + & - \\
Maihue & +++ & +++ & + \\
Rupanco & ++ & - & - \\
Llanquihue & + & + & + \\
Todos Los Santos & - & - & + \\
Chapo & - & - & + \\
\hline
\end{tabular}

Nota: Las poblaciones más estables de Stentor se encontraron en los lagos Colico, Caburgua, Pirehueico y Maihue $(+++)$. En los otros lagos se encontró solamente en algunas ocasiones $(+)$.

\section{AGRADECIMIENTOS}

Este trabajo ha sido financiado con ayuda de la Universidad Austral de Chile (DID S-200364) y el proyecto FONDECYT 1040898.

\section{BIBLIOGRAFIA}

BÜRgER, O. 1905. Estudios sobre protozoos chilenos del agua dulce. Anales de la Universidad de Chile 117: 403-449.

BÜRGER, O. 1906. Nuevos estudios sobre protozoos chilenos del agua dulce. Anales de la Universidad de Chile $118: 136-204$.

CuRDS, C.R. 1982. British and other freshwater ciliated protozoa. Cambridge University Press, Cambridge. $179 \mathrm{pp}$.

Corliss, J.O. 1979. The ciliated protozoa. Pergamon Press, Oxford. 455 pp.

DADAY, E. 1902. Beiträge zur Kenntnis der SübwasserMikrofauna von Chile. Természetrajzi Füzetek 25: 436-447.
FoISSNER W. \& S. WöLFL. 1994. Revision of the genus Stentor with the new description of Stentor araucanus $\mathrm{n}$. sp. Journal of Plankton Research 16: 255-289.

Geller, W., U. Gaedke, T. Barthelmeß, M. Lang, D. Straile \& S. WOELFL. 1996. Comparative limnology of the planktonic systems of subalpine and subandine lakes. Atti dell' 11 Congresso dell' Associazone Italiana di Oceanologia e Limnologia: 79-90.

LöffLER, H. 1961. Zur Systematik und Ökologie der chilenischen Süßwasserentomostraken. Beitrage Neotropical Fauna 2: 143-222.

Thomasson, K. 1963. Araucanian lakes. Acta Phytogeographica Sueca 47: 1-139.

WoELfL, S. 1996. Untersuchungen zur Zooplank-tonstruktur einschließlich der mikrobiellen Gruppen unter Berücksichtigung der mixotrophen Ciliaten in zwei südchilenischen Andenfußseen. Dissertation Universität Konstanz, Konstanzer Dissertationen 497, Hartung Gorre Verlag, Konstanz. 242 pp.

Woelfl, S. \& W. Geller. 2002. Chlorella - bearing ciliates dominate in an oligotrophic northpatagonian lake (Lake Pirehueico, Chile): Abundance, biomass and symbiotic photosynthesis. Freshwater Biology 47(2): 231-242.

Fecha de recepción: 19.07.05

Fecha de aceptación: 16.11.05 